\title{
Strong, tunable Terahertz emission by two-color picosecond laser irradiation
}

\author{
W.-M. Wang, ${ }^{1,2}$ P. Gibbon, ${ }^{1}$ Z.-M. Sheng, ${ }^{3,4}$ and Y.-T. Li $^{2}$ \\ ${ }^{1}$ Forschungzentrum Juelich GmbH, Institute for Advanced Simulation, \\ Juelich Supercomputing Centre, D-52425 Juelich, Germany \\ ${ }^{2}$ Beijing National Laboratory for Condensed Matter Physics, \\ Institute of Physics, CAS, Beijing 100190, China \\ ${ }^{3}$ Department of Physics, SUPA, Strathclyde University, Rottenrow 10\%, G4 0NG Glasgow, UK \\ ${ }^{4}$ Key Laboratory for Laser Plasmas (MoE) and Department of Physics and Astronomy, \\ Shanghai Jiao Tong University, Shanghai 200240, China
}

(Dated: July 18, 2014)

\begin{abstract}
It is shown by particle-in-cell simulations that powerful terahertz ( $\mathrm{THz}$ ) radiation can be generated by picosecond (ps) laser pulses below $10^{14} \mathrm{~W} / \mathrm{cm}^{2}$ via a two-color laser scheme. At such laser intensities, increasing the laser duration can result in significant enhancement in $\mathrm{THz}$ intensities. From 0.03 ps to 0.9 ps the enhancement climbs to nearly $40 \times$ before saturating until 2 ps. This demonstrates that low intensity, readily available ps laser technology could be utilized for driving powerful $\mathrm{THz}$ sources. By contrast, for laser intensities high enough to completely ionize the gas medium, it is found that the $\mathrm{THz}$ emission decreases with increasing pulse duration: optimal conversion is found for few-femtosecond drivers.
\end{abstract}

In the last two decades, $\mathrm{THz}$ radiation sources driven by advances in nonlinear optics and semiconductor technologies [1-3] have been successfully applied in diverse areas of atomic and molecular physics, surface physics, high-temperature superconductor physics, biomedicine and cellular biology. However, many other potential applications such as $\mathrm{THz}$ extreme nonlinear physics in condensed matter and nonlinear $\mathrm{THz}$ spectroscopy have remained inaccessible to date owing to the lack of reliable sources of more powerful $\mathrm{THz}$ radiation with field strengths beyond $\mathrm{MV} / \mathrm{cm}$.

High power, short-pulse laser technology offers the possibility of realizing such tabletop sources. For example, a relativistic laser pulse with intensity above $10^{18} \mathrm{~W} / \mathrm{cm}^{2}$ focused on a solid target produces powerful $\mathrm{THz}$ radiation on both the front $[4,5]$ and rear surface of the target $[6,7]$. THz radiation with energy up to $50 \mu \mathrm{J} / \mathrm{sr}$ emitted from the front surface has been measured but with a large divergence attributed to the generation mechanism via surface currents [5]. The authors of Ref. [7] reported broad-band radiation from the rear surface associated with hot electrons driving a sheath field, carrying a total energy of $460 \mu \mathrm{J}$ for incident laser intensity of $5 \times 10^{19} \mathrm{~W} / \mathrm{cm}^{2}$. With much lower intensities below $10^{16} \mathrm{~W} / \mathrm{cm}^{2}$, laser pulses passing through gases can also generate $\mathrm{THz}$ radiation [8-16]. Among the gas-target experiments, the two-color laser scheme with a fundamental pump laser mixed with its second harmonic [9-16] has shown a number of favorable properties, such as: directed emission, frequency tunability via the plasma density [8, 17-19], and importantly, high efficiency with the potential to produce $\mathrm{THz}$ emission in the multi-MV $/ \mathrm{cm}$ range. As early as $2005 \mathrm{THz}$ radiation above $0.4 \mathrm{MV} / \mathrm{cm}$ was reported [11], which is to date the strongest radiation produced by a $800-\mathrm{nm}$ pump laser pulse owing to the high intensity of $10^{17} \mathrm{~W} / \mathrm{cm}^{2}$ and the short duration of $25 \mathrm{fs}[18,20]$. A more recent experiment illustrated that the $\mathrm{THz}$ strength is considerably enhanced to 4.4
$\mathrm{MV} / \mathrm{cm}$ when the pump laser wavelength is increased to $1800 \mathrm{~nm}[16]$.

Here we propose another, simpler approach to significantly enhance the $\mathrm{THz}$ intensity by use of ps laser pulses at low intensities below $10^{14} \mathrm{~W} / \mathrm{cm}^{2}$. Compared with using longer laser wavelengths, enhancing the intensity, and shortening the duration to few cycles, this is technically easier to implement in the laboratory. At low field strengths short-pulse lasers can only ionize a small number of gas atoms. By increasing the pulse duration, more atoms will be ionized and a stronger net current along the laser field polarization will be generated. A stronger net current results in more powerful $\mathrm{THz}$ radiation. This scheme has an optimal laser duration around ps. For an even longer duration the enhancement of $\mathrm{THz}$ intensity will be saturated.

We use two-dimensional particle-in-cell (PIC) simulations to demonstrate the scheme sketched above. The PIC code KLAPS has been developed according to Refs. $[22]$, where the field ionization of atoms is included according to the Ammosov-Delone-Krainov (ADK) model $[23,24]$. With the ADK model the ionization rate of an atom is first computed in terms of the fields experienced by the atom, a probability is given according to the ionization rate, and then a Monte Carlo test is performed to determine an electron born with the probability. In the simulations, we take the lowest pump laser intensity of $5 \times 10^{13} \mathrm{~W} / \mathrm{cm}^{2}$ (the Keldysh parameter $\gamma=1.35$ ) for hydrogen and $6 \times 10^{13} \mathrm{~W} / \mathrm{cm}^{2}(\gamma=1.27)$ for nitrogen, where the Keldysh parameter is refined by $\gamma=\sqrt{\Phi / 2 U_{p}}$ [25], $\Phi$ is the ionization potential, and $U_{p}$ is the ponderomotive potential of the laser pulses. In the intermediate regime between multiphoton ionization and tunneling with $1<\gamma<8$, the ADK model can be applied [26]. At these intensities the gases start to ionize and strong $\mathrm{THz}$ radiation is generated due to the ionization which easily overcomes nonlinear optical effects in neutral gases [10].

The pump laser wavelength is fixed at $1 \mu \mathrm{m}$ and 
the second laser frequency is taken as the second harmonic of the pump one. The two laser pulses propagate along the $+\mathrm{x}$ direction. They are linearly polarized along the y direction with the electric fields $E=$ $a_{0} \exp \left(-y^{2} / r_{0}^{2}\right) \sin \left(\pi \xi / \tau_{0}\right) \times\left[\sin \left(\omega_{0} \xi\right)+\sin \left(2 \omega_{0} \xi\right) / 2\right]$ with $0 \leq \xi \leq \tau_{0}$, where $\xi=t-x / c, r_{0}=100 \mu \mathrm{m}$, and the full width at half maximum (FWHM) duration is $\tau_{0} / 2$. A gas slab with a uniform atom density of hydrogen or nitrogen of $10^{18} \mathrm{~cm}^{-3}$ is distributed between $x=300 \mu \mathrm{m}$ and $x=450 \mu \mathrm{m}$. The resolution in the $\mathrm{x}$ direction is 0.01 $\mu \mathrm{m}$. The one in the y direction is $0.1 \mu \mathrm{m}$. Initially, four simulation particles denoting gas atoms cover a cell.

Figure 1 illustrates the waveforms of the $\mathrm{THz}$ radiation propagating along the $-x$ direction in the vacuum, which emitted from the hydrogen target irradiated by the laser pulses with different intensities and FWHM durations. Here, a filter of high frequency noise has been taken to obtain the smooth waveforms, as used in Ref. [27]. From Fig.1(a) one can see that the $\mathrm{THz}$ intensity is gradually enhanced with increasing laser duration, reaching up to $34 \times$ from 30 femtosecond (fs) to 870 fs. Further increase of the duration from $870 \mathrm{fs}$ to $1200 \mathrm{fs}$, results in saturation which persists up to $1950 \mathrm{fs}$, as observed in Fig. 4(a) below. Here, we define the optimal duration $\tau_{o p t}$ as the pulse duration for which saturation of the $\mathrm{THz}$ emission occurs. This parameter decreases for increasing laser intensity, as observed in Fig. 1(b). It decreases from 870 fs to $180 \mathrm{fs}$ as the intensity is enhanced from $5 \times 10^{13} \mathrm{~W} / \mathrm{cm}^{2}$ to $10^{14} \mathrm{~W} / \mathrm{cm}^{2}$. At even higher laser intensity, a very different picture is observed: the $\mathrm{THz}$ emission is reduced for the increasing laser duration. One can see in Fig. 1(c) that the most intense $\mathrm{THz}$ radiation appears at the shortest laser duration of $5 \mathrm{fs}$, where the laser intensity is taken as $4 \times 10^{14} \mathrm{~W} / \mathrm{cm}^{2}$.

We first explain the optimal duration arising at ps level for low intensity laser pulses with the help of Figs. 2 and 3. According to Refs. [13, 17, 20], the THz intensity is proportional to the net current which is formed during the ionization. Basically, the net current is determined by the number of electrons newly released from gas atoms. Figure 2(a) shows that a small number of electrons are born within a narrow range around the laser pulse peak due to the short duration of $30 \mathrm{fs}$. By increasing the duration, the interaction time of the gas atoms with the pulses grows and the probability of the atom ionization rises. Thus, much more electrons are released in Fig. 2 (b) with the duration of $870 \mathrm{fs}$. The resulting enhancement of the net current can be clearly seen in Fig. 3(a), which shows a roughly linear scaling with the duration. Therefore, the $\mathrm{THz}$ intensity grows with the laser duration.

This growth is saturated at the duration of $870 \mathrm{fs}$ because only part of the net current has contribution to the $\mathrm{THz}$ amplitude when the duration is long enough. As presented in Refs. [17, 18], the generation mechanism of $\mathrm{THz}$ radiation is: first a net current and plasma is formed via ionization, the current drives a electrostatic (ES) oscillating field in the plasma, and then this field is transferred into electromagnetic (or $\mathrm{THz}$ ) radiation at the vacuum plasma boundaries. For a short laser duration, the net current at a given position can be considered to be formed instantly since the laser duration is much shorter than the $\mathrm{THz}$ wavelength. In this case, the whole net current at the position contributes to the radiation. While the duration at ps is comparable to or longer than the $\mathrm{THz}$ wavelength, only the part of the net current formed at earlier time has contribution to the radiation since only the first cycle can penetrate into the vacuum, as seen in Fig. 1. The effective current contributing to the radiation is plotted in Fig. 3(b) and it shows saturation starting about $870 \mathrm{fs}$, which is in agreement with the results in Figs. 1(a) and 4(a). Here we calculate the effective current with the electrons born before the radiation peak arrives at the vacuum plasma boundary. Basically, the saturation appears due to the competition of two temporal scales: the formation of the current and that of ES field in plasma. The latter is determined by the plasma density or the plasma oscillating frequency $[17,18]$. Therefore, $1 / \tau_{\text {opt }}$ is at the scale of the plasma oscillating frequency or $\mathrm{THz}$ radiation frequency.

The single cycle profile of the $\mathrm{THz}$ radiation is formed owing to the skin effect in plasma [28], which allows only the radiation source in the plasma close enough to the vacuum plasma boundaries to penetrate into the vacuum. Figure 3(c) illustrates this effect. It is shown that when the gas length is reduced to $75 \mu \mathrm{m}$ (about 0.25 radiation wavelength), the radiation is reduced in intensity compared to the one produced from the gas with the length of $600 \mu \mathrm{m}$. This suggests that the radiation source (or the net current) $150 \mu \mathrm{m}$ far away the boundary has no effect on the radiation observed in vacuum. Here, we take the $\mathrm{THz}$ radiation propagating along the $-\mathrm{x}$ direction, which is not sensitive to the gas length if it is larger than the radiation wavelength, as just displayed in Fig. 3(c). However, the radiation along the $+\mathrm{x}$ direction co-propagates with the laser pulses in gas plasma and therefore depends on the gas length due to absorption and propagation effects.

Next we explain appearance of the radiation peak at few-fs laser pulses, as shown in Fig. 1(c). Since the THz intensity is proportional to the net current, it depends on both the number of the electrons born and their net velocities. The latter is linearly proportional to the laser vector potential value at the position where an electron is born [17, 21]. If the electron is born at the laser pulse peak, it will gain a large net velocity. Otherwise, it will get small one if it is born away from the pulse peak. At $4 \times 10^{14} \mathrm{~W} / \mathrm{cm}^{2}$, the $5 \mathrm{fs}$ pulses ionize the gas atoms completely and the electrons are born around the pulse peak, as seen in Fig. 2(c). As the duration is increased to $90 \mathrm{fs}$, the leading edge of the pulses can ionize all of the atoms and therefore the electrons are observed to be born away from the pulse peak in Fig. 2(d). Their net velocities gained from the $90 \mathrm{fs}$ pulses are smaller than the 5 fs ones while the electron number is the same in the two cases due to complete ionization. Hence, the net 
current and the $\mathrm{THz}$ radiation is stronger for the shorter pulses.

Note that in Fig. 1 the $\mathrm{THz}$ duration (or equivalently wavelength for this single cycle emission) decreases with the increasing laser intensity; it also reduces with the increasing laser duration at a given low laser intensity, as shown in Figs. 1(a) and 1(b), but stays almost unchanged at a high laser intensity, as seen in Fig. 1(c). The reason is as follows. The central frequency of the $\mathrm{THz}$ radiation is proportional to the plasma density [8, 17, 18]. Obviously the plasma density grows with the increasing laser intensity and duration. However, once the gas is ionized completely at a high enough laser intensity, the density will remain clamped to its maximum possible value.

Figures 4 (a) and 4(b) display the $\mathrm{THz}$ intensity as a function of the laser duration and Fig. 4(c) shows the optimal pulse durations corresponding to different laser intensities. More clearly than Figs. 1(a) and 1(b), Fig. 4(a) illustrates that the saturation regions start from an optimal duration in the ps range and remain a large range of durations at low intensities, at which part ionization of gas arises. At $5 \times 10^{13} \mathrm{~W} / \mathrm{cm}^{2}$ the saturation occurs between 870 fs and $1950 \mathrm{fs}$; at $6 \times 10^{13} \mathrm{~W} / \mathrm{cm}^{2}$ it between 510 fs and 900 fs. Therefore, one may take any pulse duration within these plateau regions to obtain the strongest $\mathrm{THz}$ emission. One can also see that the normalized intensity of the $\mathrm{THz}$ radiation at the optimal durations reduces with increasing laser intensity. From Fig. 4(c) one can observe that the optimal duration goes down with the intensity. The two results can be explained as: at a lower laser intensity, a smaller part of gas atoms are ionized with a short laser duration, say $30 \mathrm{fs}$, and there is a larger duration range to reach the optimal duration. One can also see in Fig. 4(b) more clearly than Fig. 1(c) that the $\mathrm{THz}$ intensity reduces with the increasing laser duration at high intensities, where complete ionization dominates.

So far, we have considered hydrogen targets. To test a more complex case we also present results for nitrogen in Fig. 5. Like hydrogen, it has a low first ionization potential so laser pulses with very low intensities can be used to ionize the gas and to produce $\mathrm{THz}$ radiation. Similar results to the case with the hydrogen can be seen in this figure. It is interesting to note the duration range for the saturation region is as follows: at $6 \times 10^{13} \mathrm{~W} / \mathrm{cm}^{2}$ the plateau appears within $660-1800 \mathrm{fs}$ and at $10^{14} \mathrm{~W} / \mathrm{cm}^{2}$ it within 240-540 fs. One notices that at the same laser intensity the optimal duration is longer in the nitrogen and the normalized $\mathrm{THz}$ intensity is higher. This is because nitrogen atoms have a higher first ionization potential and a smaller part of atoms are ionized at the same laser parameters. It should be pointed out that the $\mathrm{THz}$ intensities are obtained with $r_{0}=100 \mu \mathrm{m}$ and they change slightly when $r_{0}$ is increased even to infinite, i.e., a plane wave.

We note that there has been an experimental study on laser pulse duration dependent $\mathrm{THz}$ emission [29]. It shows that an intensity peak of forward $\mathrm{THz}$ signals ap- pears at 150 fs when the pulse duration is changed between 42 fs and 240 fs with a given laser energy. However, no saturation has been observed. This radiation enhancement was explained by the competition and cooperation of multiple filaments in long distance propagation of the laser pulse with strong defocusing since the laser power was taken to be much above the critical power. This explanation is also supported by the fact that positively chirped laser pulses generate more powerful radiation than negatively chirped pulses with the same duration. In addition, coherence and/or incoherence addition of the $\mathrm{THz}$ signals producing at different positions along the laser propagation may also contribute to the result [30]. In our case, $\mathrm{THz}$ radiation is measured in the backward direction, which is determined by the initial laser parameters. Therefore the physical mechanism in our scheme is different.

TABLE I. The amplitudes $(\mathrm{MV} / \mathrm{cm})$ and central frequencies of $\mathrm{THz}$ radiation produced by the lasers with different pump laser intensities and FWHM durations, as shown in the first two rows. The first column is atom densities of hydrogen. The last row is energy conversion efficiency $\eta$ from the laser pulses to the THz radiation with the atom density $2 \times 10^{19} \mathrm{~cm}^{-3}$.

\begin{tabular}{llll}
\hline \hline & $5 \times 10^{13} \mathrm{~W} / \mathrm{cm}^{2}$ & $6 \times 10^{13} \mathrm{~W} / \mathrm{cm}^{2}$ & $10^{14} \mathrm{~W} / \mathrm{cm}^{2}$ \\
& $870 \mathrm{fs}$ & $510 \mathrm{fs}$ & $180 \mathrm{fs}$ \\
\hline $1 \times 10^{18} \mathrm{~cm}^{-3}$ & $0.20 @ 1.1 \mathrm{THz}$ & $0.43 @ 1.5 \mathrm{THz}$ & $1.2 @ 3.1 \mathrm{THz}$ \\
$5 \times 10^{18} \mathrm{~cm}^{-3}$ & $0.34 @ 1.3 \mathrm{THz}$ & $0.54 @ 2.0 \mathrm{THz}$ & $1.5 @ 4.6 \mathrm{THz}$ \\
$1 \times 10^{19} \mathrm{~cm}^{-3}$ & $0.40 @ 1.4 \mathrm{THz}$ & $0.66 @ 2.4 \mathrm{THz}$ & $1.9 @ 5.1 \mathrm{THz}$ \\
$2 \times 10^{19} \mathrm{~cm}^{-3}$ & $0.48 @ 1.5 \mathrm{THz}$ & $0.82 @ 2.7 \mathrm{THz}$ & $2.4 @ 7.1 \mathrm{THz}$ \\
$\eta$ & $2.3 \times 10^{-6}$ & $5.4 \times 10^{-6}$ & $3.0 \times 10^{-5}$ \\
\hline \hline
\end{tabular}

TABLE II. The amplitudes (MV/cm) and central frequencies of $\mathrm{THz}$ radiation produced by the lasers with different pump laser intensities and FWHM durations, as shown in the first two rows. The first column is atom densities of nitrogen. The last row is energy conversion efficiency $\eta$ from the laser pulses to the $\mathrm{THz}$ radiation with the atom density $2 \times 10^{19} \mathrm{~cm}^{-3}$.

\begin{tabular}{lll}
\hline \hline & $6 \times 10^{13} \mathrm{~W} / \mathrm{cm}^{2}$ & $10^{14} \mathrm{~W} / \mathrm{cm}^{2}$ \\
& $660 \mathrm{fs}$ & $240 \mathrm{fs}$ \\
\hline $1 \times 10^{18} \mathrm{~cm}^{-3}$ & $0.19 @ 0.9 \mathrm{THz}$ & $0.6 @ 2.4 \mathrm{THz}$ \\
$5 \times 10^{18} \mathrm{~cm}^{-3}$ & $0.35 @ 1.5 \mathrm{THz}$ & $1.1 @ 3.9 \mathrm{THz}$ \\
$1 \times 10^{19} \mathrm{~cm}^{-3}$ & $0.43 @ 1.8 \mathrm{THz}$ & $1.4 @ 4.1 \mathrm{THz}$ \\
$2 \times 10^{19} \mathrm{~cm}^{-3}$ & $0.58 @ 2.1 \mathrm{THz}$ & $1.9 @ 4.6 \mathrm{THz}$ \\
$\eta$ & $2.7 \times 10^{-6}$ & $2.2 \times 10^{-5}$ \\
\hline \hline
\end{tabular}

Tables I and II summarize the amplitudes $(\mathrm{MV} / \mathrm{cm})$ and frequencies of $\mathrm{THz}$ emission from hydrogen and nitrogen gases, respectively, which may be referred by experiments. The first two rows show the pump laser intensities and the durations used in the simulations, where the duration values are the corresponding $\tau_{o p t}$ when the atom density is taken as $10^{18} \mathrm{~cm}^{-3}$. We take different atom densities shown in the first column. With the increase of the density, the laser pulses interacting with more atoms produce a larger number of electrons, which 
results in stronger $\mathrm{THz}$ emission. The density of produced plasma also rises and consequently the $\mathrm{THz}$ frequency goes up. Meanwhile, $\tau_{\text {opt }}$ lowers because it depends on the $\mathrm{THz}$ wavelength and thus inversely on the density. This means that the duration value used in the simulation is higher than $\tau_{\text {opt }}$ when the density is above $10^{18} \mathrm{~cm}^{-3}$, which is within the saturation plateau. From Tab. I one can see that the $\mathrm{THz}$ amplitude is up to 0.48 $\mathrm{MV} / \mathrm{cm}$ at $5 \times 10^{13} \mathrm{~W} / \mathrm{cm}^{2}$ laser intensity; it reaches a value as high as $2.4 \mathrm{MV} / \mathrm{cm}$ at $10^{14} \mathrm{~W} / \mathrm{cm}^{2}$. Note that these $\mathrm{THz}$ amplitudes can be achieved with any laser duration within a large range after saturation.

It is interesting to compare the possible highest radiation amplitudes generated by ps and fs laser pulses. We fix the two groups of laser parameters as: one is with 870 fs and $5 \times 10^{13} \mathrm{~W} / \mathrm{cm}^{2}$ and another with $30 \mathrm{fs}$ and $4 \times 10^{14} \mathrm{~W} / \mathrm{cm}^{2}$. As the radiation frequency depends upon the plasma density, we change the hydrogen density to get the radiation at the same frequency for the two cases. According to Ref. [21], the radiation amplitude is saturated when the pulse intensity exceeds $3 \times 10^{14} \mathrm{~W} / \mathrm{cm}^{2}$ with the $30 \mathrm{fs}$ pulses. Our simulation shows the highest amplitude is $0.26 \mathrm{MV} / \mathrm{cm}$ with the $30 \mathrm{fs}$ pulses when the radiation frequency is at $1.5 \mathrm{THz}$. The corresponding value is $0.48 \mathrm{MV} / \mathrm{cm}$ with the 870 fs pulses as given in Table I. Similarly, when the radiation frequency is enhanced to $7.1 \mathrm{THz}$ by increasing the hydrogen density, its highest amplitude is $1.23 \mathrm{MV} / \mathrm{cm}$ with the $30 \mathrm{fs}$ pulses while it reaches $2.4 \mathrm{MV} / \mathrm{cm}$ with the 180 fs pulses at
$10^{14} \mathrm{~W} / \mathrm{cm}^{2}$ as presented in Table I. These examples suggest that the saturation amplitude with ps pulses is even higher than that with fs pulses.

In summary, we have demonstrated the potential of ps laser pulses at low intensities for generation of powerful $\mathrm{THz}$ radiation, in contrast to previous studies which have concentrated on laser pulses with tens of fs or below. At low intensities the laser pulses with long durations of ps show distinct advantages over the fs ones. For example, the $\mathrm{THz}$ radiation generated by the $5 \times 10^{13} \mathrm{~W} / \mathrm{cm}^{2}$ pulses of $0.9 \mathrm{ps}-2 \mathrm{ps}$ is nearly 40 times stronger than that by the pulses of $30 \mathrm{fs}$. In particular, the pulses at $10^{14} \mathrm{~W} / \mathrm{cm}^{2}$ can produce $\mathrm{THz}$ radiation of a few $\mathrm{MV} / \mathrm{cm}$. However, such advantages of long duration pulses are eroded with increasing laser intensity because of ionization quenching. When the intensity is high enough to ionize the gas completely, a very short duration, e.g., 5 fs, may be more favorable.

\section{ACKNOWLEDGMENTS}

Computing resources were provided through the FZJülich VSR grant number JZAM04. This work was supported by the Alexander von Humboldt Foundation, 973 Program (Grant No. 2013CBA01501 and 2014CB339800), and NSFC (Grant Nos. 11375261, 11105217, and 11121504).
[1] M. Bass, P. A. Franken, J. F. Ward, and G. Weinreich, Phys. Rev. Lett. 9, 446 (1962).

[2] A. Nahata, A. S. Welling, and T. F. Heinz, Appl. Phys. Lett. 69, 2321 (1996).

[3] A. J. Taylor, P. K. Benicewicz, S. M. Young, Opt. Lett. 18, 1340 (1993).

[4] C. Li, M.-L. Zhou, W.-J. Ding, F. Du, F. Liu, Y.-T. Li, W.-M. Wang, Z.-M. Sheng, J.-L. Ma, L.-M. Chen, X. Lu, Q.-L. Dong, Z.-H. Wang, Z. Lou, S.-C. Shi, Z.-Y. Wei, and J. Zhang, Phys. Rev. E 84, 036405 (2011).

[5] Y. T. Li, C. Li, M. L. Zhou, W. M. Wang, F. Du, W. J. Ding, X. X. Lin, F. Liu, Z. M. Sheng, X. Y. Peng, L. M. Chen, J. L. Ma, X. Lu, Z. H. Wang, Z. Y. Wei, and J. Zhang, Appl. Phys. Lett. 100, 254101 (2012).

[6] A Gopal, T May, S Herzer, A Reinhard, S Minardi, M Schubert, U Dillner, B Pradarutti, J Polz, T Gaumnitz, M C Kaluza, O Jackel, S Riehemann, W Ziegler, H-P Gemuend, H-G Meyer and G G Paulus, New J. Phys. 14, 083012 (2012).

[7] A. Gopal, S. Herzer, A. Schmidt, P. Singh, A. Reinhard, W. Ziegler, D. Brommel, A. Karmakar, P. Gibbon, U. Dillner, T. May, H-G. Meyer, and G. G. Paulus, Phys. Rev. Lett. 111, 074802 (2013).

[8] H. Hamster, A. Sullivan, S. Gordon, W. White, and R. W. Falcone, Phys. Rev. Lett. 71, 2725 (1993).

[9] D. J. Cook and R. M. Hochstrasser, Opt. Lett. 25, 1210 (2000).

[10] M. Kress, T. Loffler, S. Eden, M. Thomson, and H. G.
Roskos, Opt. Lett. 29, 1120 (2004).

[11] T. Bartel, P. Gaal, K. Reimann, M. Woerner, and T. Elsaesser, Opt. Lett. 30, 2805 (2005).

[12] X. Xie, J. Dai, and X.-C. Zhang, Phys. Rev. Lett. 96, 075005 (2006).

[13] K. Y. Kim, J. H. Glownia, A. J. Taylor and G. Rodriguez, Opt. Express 15, 4577 (2007).

[14] Y. S. You, T. I. Oh, and K. Y. Kim, Phys. Rev. Lett. 109, 183902 (2012).

[15] X.-Y. Peng, R. Jung, T. Toncian, O. Willi, and J.-Hu. Teng, Appl. Phys. Lett. 94, 221107 (2009).

[16] M. Clerici, M. Peccianti, B. E. Schmidt, L. Caspani, M. Shalaby, M. Giguere, A. Lotti, A. Couairon, F. Legare, T. Ozaki, D. Faccio, and R. Morandotti, Phys. Rev. Lett. 110, 253901 (2013).

[17] W.-M. Wang, Z.-M. Sheng, H.-C. Wu, M. Chen, C. Li, J. Zhang, and K. Mima, Opt. Express 16, 16999 (2008).

[18] W.-M. Wang, S. Kawata, Z.-M. Sheng, Y.-T. Li, J. Zhang, Phys. Plasmas 18, 073108 (2011)

[19] M. Chen, A. Pukhov, X.-Y. Peng, and O. Willi, Phys. Rev. E 78, 046406 (2008).

[20] H. C. Wu, J. Meyer-ter-Vehn, and Z. M. Sheng, New J. Phys. 10, 043001 (2008).

[21] W.-M. Wang, Y.-T. Li, Z.-M. Sheng, X. Lu, and J. Zhang, Phys. Rev. E 87, 033108 (2013).

[22] C. K. Birdsall and A. B. Langdon, Plasma Physics via Computer Simulation (McGraw-Hill, New York, 1985).

[23] M. V. Ammosov, N. B. Delone, and V. P. Krainov, Sov. 
Phys. JETP 64, 1191 (1986).

[24] B. M. Penetrante and J. N. Bardsley, Phys. Rev. A 43, 3100 (1991).

[25] L. V Keldysh, Soy. Phys. JETP, 20, 1307 (1965).

[26] G. Gibson, T. S. Luk, and C. K. Rhodes, Phys. Rev. A 41, 5049 (1990).

[27] W.-M. Wang, S. Kawata, Z.-M. Sheng, Y.-T. Li, L. M. Chen, L. J. Qian, J. Zhang, Opt. Lett. 36, 2608 (2011).

[28] P. Gibbon, Short Pulse Laser Interactions with Matter (Imperial College Press, 2000).

[29] T.-J. Wang,Y. Chen, C. Marceau, F. Theberge, M. Chateauneuf, J. Dubois, and S. L. Chin, Appl. Phys. Lett. 95, 131108 (2009).

[30] Y. Liu, A. Houard, M. Durand, B. Prade, and A. Mysyrowicz, Opt. Express 17, 11480 (2009).

\section{Figure Captions}

Fig. 1. (Color online) $\mathrm{THz}$ radiation on the axis $(y=0)$. Different curves correspond to different laser FWHM durations, where the $\mathrm{THz}$ intensities are normalized by the 30 fs case, i.e., $I_{30 f s}=1.7 \times 10^{6} \mathrm{~W} / \mathrm{cm}^{2}$, $2.0 \times 10^{8} \mathrm{~W} / \mathrm{cm}^{2}$, and $2.7 \times 10^{9} \mathrm{~W} / \mathrm{cm}^{2}$, respectively. In (a)-(c) the pump laser intensities are $5 \times 10^{13} \mathrm{~W} / \mathrm{cm}^{2}$, $10^{14} \mathrm{~W} / \mathrm{cm}^{2}$, and $4 \times 10^{14} \mathrm{~W} / \mathrm{cm}^{2}$, respectively.

Fig. 2. (Color online) The number distributions of electrons born from gas atoms versus their birth locations in the reference frame co-moving with the laser, where $t$ and $x$ are the birth time and positions and the origin denotes the laser pulse center. (a) and (b) are the results with the laser intensity of $5 \times 10^{13} \mathrm{~W} / \mathrm{cm}^{2}$ and the FWHM durations of 30 fs and 870 fs, respectively. (c) and (d) are the ones with the intensity of $4 \times 10^{14} \mathrm{~W} / \mathrm{cm}^{2}$ and the FWHM durations of $5 \mathrm{fs}$ and $90 \mathrm{fs}$, respectively.

Fig. 3. (Color online) The total current (a) and the effective current (b) as a function of the laser FWHM duration. (c) is $\mathrm{THz}$ radiation on the axis $(\mathrm{y}=0)$, where different curves correspond to the cases with different lengths of gas targets and the laser intensity $5 \times 10^{13} \mathrm{~W} / \mathrm{cm}^{2}$ and duration $870 \mathrm{fs}$ is taken. The units of the currents in (a) and (b) and the $\mathrm{THz}$ intensities in (c) are taken as the ones with 30 fs laser duration.

Fig. 4. (Color online) (a) and (b) are $\mathrm{THz}$ intensities versus laser FWHM durations, where the intensities are normalized by the one with 30 fs laser duration, i.e., $I_{30 f s}=1.7 \times 10^{6} \mathrm{~W} / \mathrm{cm}^{2}, 6.1 \times 10^{6} \mathrm{~W} / \mathrm{cm}^{2}$, $2.2 \times 10^{9} \mathrm{~W} / \mathrm{cm}^{2}$ and $2.7 \times 10^{9} \mathrm{~W} / \mathrm{cm}^{2}$, for different pump laser intensities $5 \times 10^{13} \mathrm{~W} / \mathrm{cm}^{2}, 6 \times 10^{13} \mathrm{~W} / \mathrm{cm}^{2}$, $2 \times 10^{14} \mathrm{~W} / \mathrm{cm}^{2}$, and $4 \times 10^{14} \mathrm{~W} / \mathrm{cm}^{2}$, respectively. (c) is the optimal duration as a function of the pump laser intensity. Here hydrogen is taken.

Fig. 5. (Color online) (a) and (b) are $\mathrm{THz}$ intensities versus laser FWHM durations, where the intensities are normalized by the one with 30 fs laser duration, i.e., $I_{30 f s}=1.3 \times 10^{6} \mathrm{~W} / \mathrm{cm}^{2}, 7.5 \times 10^{7} \mathrm{~W} / \mathrm{cm}^{2}$, $1.8 \times 10^{9} \mathrm{~W} / \mathrm{cm}^{2}$ and $3.2 \times 10^{9} \mathrm{~W} / \mathrm{cm}^{2}$, for different pump laser intensities $6 \times 10^{13} \mathrm{~W} / \mathrm{cm}^{2}, 10^{14} \mathrm{~W} / \mathrm{cm}^{2}$, $2 \times 10^{14} \mathrm{~W} / \mathrm{cm}^{2}$, and $4 \times 10^{14} \mathrm{~W} / \mathrm{cm}^{2}$, respectively. (c) is the optimal duration as a function of the pump laser intensity. Here nitrogen is taken. 